\title{
Depresión, recursos
}

psicológicos y

vulnerabilidad cognitiva a la

\section{depresión en jóvenes \\ estudiantes universitarios}

Alicia Edith Hermosillo de la Torre ${ }^{1}$ y María Elena Rivera Heredia ${ }^{2}$

\section{Resumen}

Desde el paradigma diátesis-estrés la depresión es concebida como el resultado de la interacción entre eventos vitales estresantes (estrés) y factores cognitivos aprendidos que confieren vulnerabilidad a un individuo (diátesis). La teoría de la depresión por desesperanza postula la existencia de un estilo atribucional depresivo que se desarrolla en los primeros años

1 Universidad Autónoma de Aguascalientes, alicia.hermosillo@edu.uaa.mx

2 Universidad Michoacana de San Nicolás de Hidalgo 
de vida y confiere vulnerabilidad cognitiva a la depresión en la edad adulta. Dicha vulnerabilidad incrementa el riesgo de presentar depresión en ausencia de factores de protección por lo que resulta importante analizar la forma en que se asocian durante la juventud. El presente estudio analiza la relación entre los recursos psicológicos y la vulnerabilidad cognitiva a la depresión así como su interacción con la sintomatología depresiva y depresión en jóvenes universitarios. Participaron 93 estudiantes universitarios entre 18 y 25 años de edad (54.8\% mujeres, 45.2\% hombres). Se utilizaron las siguientes instrumentos: CES-DR adaptada para adolescentes mexicanos ( $\alpha=.93)$; Cuestionario de Vulnerabilidad Cognitiva (CSQ-UAA) adaptado para jóvenes de Aguascalientes $(\alpha=.91)$; Escala de Calidad de la Red ( $\alpha=.91)$; Escalas de Recursos Psicológicos ( $\alpha=.91)$; y la entrevista MINI 2.0., en espa-ñol. Se observaron diferencias significativas en la dificultad para solicitar apoyo y para manejar la tristeza entre los sujetos con VCD $(p=.000)$. Sintomatología depresiva se asoció positivamente con desesperanza $(r=.696, p \leq .05)$ y VCD $(r=204, p \leq .05)$; la calidad de red de amigos correlacionó negativamente con red de apoyo y autocontrol mientras que dificultad para solicitar apoyo y manejar la tristeza se asociaron positivamente con VCD. Los resultados sugieren que el apoyo social y los recursos psicológicos tienen un efecto moderador sobre la VCD y la sintomatología depresiva, 
no obstante el apoyo social no necesariamente es un factor que contribuye a reducir los síntomas depresivos.

Palabras clave: vulnerabilidad cognitiva a la depresión; recursos psicológicos; depresión por desesperanza; adultez temprana; depresión.

\section{Abstract}

From the diathesis-stress paradigm, depression is conceived as the result of the interaction between stressful life events (stress) and learned cognitive factors that confer vulnerability to an individual (diathesis). The Hopelessness Depression Theory postulates develop of a depressive attributional style in the first years of life that confers cognitive vulnerability to depression in adulthood. This vulnerability increases the risk of presenting depression in the absence of protective factors, so it is important to analyze the way they are associated during youth. The present study analyzes the relationship between psychological resources and cognitive vulnerability to depression as well as their interaction with depressive symptomatology and depression in university students. Participants were 93 university students between 18 and 25 years of age (54.8\% women, $45.2 \%$ men). The following instruments were used: CES-DR adapted for Mexican adolescents $(\alpha=.93)$; Cognitive Vulnerability Questionnaire (CSQ-UAA) adapted for Aguascalientes youth $(\alpha=.91)$; Network Quality Scale $(\alpha=.91)$; Scales 
of Psychological Resources $(\alpha=.91)$; and the MINI 2.0 interview, in Spanish. Significant differences were observed in the difficulty to request support and to manage sadness among subjects with VCD $(p=.000)$. Depressive symptomatology was positively associated with hopelessness $(r=.696, p \leq .05)$ and VCD $(r=204$, $p \leq .05)$; the network quality of friends negatively correlated with support network and self-control while difficulty in requesting Support and manage sadness were positively associated with VCD. The results suggest that social support and psychological resources have a moderating effect on VCD and depressive symptomatology, although social support is not necessarily a factor that contributes to reducing depressive symptoms.

Keywords: cognitive vulnerability to depression; psychological resources; depression hopelessness theory; early adulthood; depression.

La depresión es un problema severo que afecta a más de 300 millones de personas en el mundo y es la principal causante de años de vida perdidos por discapacidad (APD $=7.5 \%$ en el año 2015), así como la principal afección que contribuye a las muertes por suicidio en el mundo (OMS, 2017). Los altos costes sociales y laborales asociados a esta problemática, y alta prevalencia en adolescentes y jóvenes en nuestro país y el mundo, así como su relación con las conductas suicidas hace necesaria una mayor 
comprensión del problema (Benjet, Borjes, Méndez, Albor, Cassanova, Orozco et al., 2016; Benjet, Borges, MedinaMora, Martínez, Herrmosillo-De la Torre, et al., 2016; Borges, Benjet, Medina-Mora, Orozco, Familiar, Nock et al., 2010; Ferrari, Norman, Freedman, Baxter, Pirkis, Harris \& Whiteford, 2014.

La teoría de la depresión por desesperanza ha mostrado su validez y utilidad clínica en la predicción de la depresión con adolescentes y adultos jóvnes (Mac Giollabhui, Hamilton, Nielsen, Connolly, Stange, Varga, Burdette, Olino, Abramson, \& Alloy, 2018). Tal aproximación postula que la depresión es causada por estilos cognitivos mal adaptativos caracterizados por actitudes disfuncionales del "sí", tendencia a la realización de inferencias negativas acerca de las causas y consecuencias de eventos negativos, así como la tendencia a rumiar acerca de los propios sentimientos de tristeza. Estos estilos cognitivos confieren a los individuos estructuras cognitivas vulnerables a la depresión que bajo condiciones contextuales de alto estrés desembocan en estados de desesperanza que causan la depresión.

La vulnerabilidad cognitiva a la depresión se ha abordado en diversos estudios con población joven en contextos universitario (Beiter, Nash, McCrady, Rhoa-des, Linscomb, Clarahan, \& Sammut, 2015; Xiao, Qiu, He, Cui, Auerbach, McWhinnie, \& Yao, 2016). Se ha encontrado que la vulnerabilidad cognitiva a la depresión se asocia con la presencia de síntomas depresivos, depresión (Fresco, 2006; Alloy, 2006; Hanking, 2005; Herrera, 2001) e intentos de suicidio (O'Connor, 2000). 
No obstante lo anterior, se tiene evidencia que los efectos nocivo de los esquemas cognitivos vulnerables a la depresión son inhibidos por la presencia de factores protectores de orden psicosocial también llamados recursos psicológicos, específicamente se ha observado que el apoyo social disminuye el riesgo de depresión a través de la mediación sobre la deses-peranza (Johnson, Alloy, Panzarella, Metalsky, Rabkin, Williams, \& Abramson, 2001). Por otra parte, Rivera-Heredia y Andrade (2006) reportaron el efecto positivo de recursos psicológicos de manejo en el autocontrol y la recuperación del equilibrio en los adolescentes sin síntomas depresivos e intento de suicidio en contraste con adolescentes con intento de suicidio, de igual forma, observaron en los primeros una mayor capacidad para solicitar apoyo y mejores relaciones familiares.

El presente estudio tiene por objetivo analizar las relaciones dadas entre el apoyo social y los recursos psicológicos, y la vulnerabilidad cognitiva a la depre-sión, la desesperanza y la sintomatología depresiva con el propósito de identificar la participación de estos recursos en futuros modelos explicativos de la depresión.

\section{Método}

\section{Participantes}

De un total de 334 estudiantes universitarios de la Universidad Autónoma de Aguascalientes elegidos por muestreo aleatorio estratificado para el estudio deno- 
minado "El apoyo social y los recursos psicológicos como predictores de la Vulnerabilidad Cognitiva a la Depresión", para este estudio participaron 93 estudiantes que mostraron interés por acudir a la evaluación. Del total de los sujetos que participaron, el $54.8 \%$ fueron del género femenino y $45.2 \%$ del género masculino con un rango de edad que osciló entre los 18 y 26 años $(M=21.6, D E=1.67) ; 96.7 \%$ eran solteros, el resto estaba casado, vivía en unión libre o se había separado; el $39.8 \%$ se dedicaba solamente a estudiar mientras que, casi la mitad de los estudiantes, el $48.4 \%$ se dedicaban a estudiar y trabajar mientras que el $10.8 \%$ además de estudiar y trabajar se encargaban de labores domésticas.

Instrumentos

Escala de Depresión del Center of Epidemiological Studies (CES-D). Se utilizó la versión CES-D-R valida-da para estudiantes mexicanos por González-Forteza, Jimenez-Tapia, Ramos-Lira y Wagner (2008) la cual muestra una consistencia interna global alta $(\alpha=.93)$. El instrumento permite valorar la presencia y frecuencia de los síntomas depresivos en cinco categorías: a) sin relevancia clínica; b) depresión sub-umbral; c) probable episodio depresivo mayor (DSM-IV); d) altamente probable episodio depresivo mayor (DSM-IV); y e) episodio depresivo mayor. Está conformado por 35 reactivos cuyas respuestas se ubican en una escala Likert 
de 5 puntos que van de 0 (escasamente, 0 a 1 días) a 4(casi diario durante las últimas dos semanas, de 10 a 14 días) en función de los días en que se ha presentado el síntoma en cuestión. Los reactivos 2, 4, 6, 21, 24, 26, 27, 32, y 34 evalúan disforia; el 8, 10, 31, y 33 corresponden a anhedonia; el 1, y 18, cambios en el apetito; 5, 11, y 19, problemas con el sueño; 12 y 13 agitación/retardo psicomotor; 7, 16 y 23, fatiga; 9, 17, 22 y 25, culpa excesiva o inapropiada; 3 y 20, pensamiento; 14 y 15 ideación suicida; y los ítems 28, 29, 30 y 35, evalúan amigos/ocupación/familia. En este estudio la escala de desesperanza medida obtuvo un alfa muy baja $(\alpha=.298)$ pero esta mejoró eliminando el ítem $24(\alpha=.62)$ por lo que desesperanza se calculó con los ítems 24 y 34.

Cuestionario de Estilos Cognitivos (CSQ-RM versión corta, revisión mexicana). El CSQ-RM es un cuestionario de auto-reporte adaptado del CSQ (Abramson, Metalsky \& Alloy, 1989) para población mexicana ${ }^{3}$. En el marco del modelo de depresión por desesperanza, este instrumento mide la vulnerabilidad cognitiva a la depresión (VCD), entendida como la tendencia que tiene un individuo a atribuir causas globales y estables a eventos vitales negativos considerando que éstos traerán otras consecuencias negati-

3 Adaptado por los miembros del Cuerpo Académico Desarrollo Psicológico, Cultura y Salud del Depto. de Psicología de la Universidad Autónoma de Aguascalientes. Este cuestionario ha sido traducido y adaptado al español del CSQ (Haeffel, Gibb, Metalsky, Alloy, Abramson, Hankin, Joiner Jr., \& Swendsen, 2007) por el Mtro. Armando Villalobos Nájera, la Lic. Mayra Alejandra Torres Guzmán y la Mtra. Alicia Edith Hermosillo de la Torre, con permiso de los autores. 
vas en su vida e implicándose en dichos eventos como una persona de poca valía. Esta versión está conformada por 14 viñetas que abarcan los dominios de logro y relaciones interpersonales en jóvenes que estudian la universidad. Diez de las catorce situaciones se refieren a eventos negativos y son las que miden la vulnerabilidad cognitiva a la depresión. Cada situación hipotética es evaluada mediante 6 preguntas, la primera es abierta y las restantes son de formato Likert con respuesta graduada en 7 puntos. Los tres componentes que constituyen el factor de vulnerabilidad cognitiva de la depresión: a) atribuciones causales; b) consecuencias; y c) características de autovaloración son evaluados a partir de la pregunta 3 de cada situación. El instrumento ha mostrado tener una buena consistencia con estudiantes universitarios de Aguascalientes teniendo un alfa de Cronbach de .91 en el global y de .82, .82 y .88 en las subdimensiones causalidad, consecuencias y autovaloración respectivamente. El análisis de estructura factorial muestra que los componentes de la vulnerabilidad cognitiva tienden a agruparse en distintos factores sugiriendo una aceptable validez empírica en dichos componentes. El cuestionario puede ser administrado de forma individual y colectiva y tiene una duración de 20 a 30 minutos.

Escala de Calidad de la Red, ECAR (Nava, Reyes-Lagunes, \& Vega, 2001). Escala de auto reporte que evalúa la red de apoyo social en tres factores: a) unión familiar, b) amistad y confianza y c) vecinos y 
compañeros. Para fines de este estudio solo se han tomado los factores b y c. La escala en su conjunto explica el $48.1 \%$ de la varianza y se conforma por un total de 35 reactivos con una escala de respuesta Likert de cinco niveles que van desde muy de acuerdo (1) hasta muy en desacuerdo (5). Los ítems 23, 2, 34 , $29,32,26,17,4,14,10,24,20$, y 5 evalúan el factor amistad y confianza; mientras que los ítems 15, 21, 30, 9, 27 y 13, evalúan el factor vecinos y compañeros.

Escalas de Recursos Psicológicos, ERP (RiveraHeredia \& Andrade, 2006). El instrumento está compuesto por cinco escalas que en conjunto evalúan los elementos internos o externos con los que cuenta una persona para encarar las diferentes situaciones que se le presentan a través de cinco escalas: recursos afectivos, cognitivos, instrumentales, sociales y mate-riales. Para fines de este estudio se aplicaron dos de las cinco escalas de Recursos Psicológicos, en este caso las de recursos afectivos $(\alpha=.82)$ y sociales $(\alpha=.75)$. El formato de respuesta es Likert de 4 puntos que van de 4 (casi siempre) a 1 (casi nunca). La escala se califica obteniendo el puntaje total mediante la suma de los valores asignados en los reactivos de cada una de las escalas una vez habiendo invertido los valores de los reactivos 2, 4, 40, 43, y 44. Posteriormente se calcula el puntaje promedio dividiendo el puntaje total entre el número de reactivos que componen la subescala. 
Procedimiento

Los participantes fueron contactados telefónicamente e invitados a participar en forma voluntaria en el estudio una vez que fueron informados sobre los objetivos y fines del mismo. Los instrumentos se aplicaron en forma colectiva y en formato de lápiz y papel en las instalaciones de la Unidad de Atención e Investigación en Psicología del Departamento de Psicología en la Universidad Autónoma de Aguascalientes. Los casos que fueron identificados con depresión fueron canalizados a recibir atención psicológica en la Clínica de Psicología de la Institución.

Análisis

Los datos fueron procesados con la ayuda del SPSS 17. Se calcularon cuartiles para identificar los grupos con baja/alta VCD así como el grupo sin VCD. Se obtuvieron las categorías de significancia clínica para EDM así como la sub-escala de desesperanza con la ayuda del algoritmo de Eaton et al., (1998) y adaptado por Wagner (1999). Se analizó la relación entre las variables de estudio mediante la prueba de correlación de Pearson con la finalidad de observar el tipo y dirección de las correlaciones estadísticamente significativas. Para el análisis de las diferencias entre los grupos con y sin VCD se hicieron comparaciones de las categorías clínicas de la CES-DR, desesperanza, sintomatología depresiva, apoyo social y recursos 
psicológicos mediante un análisis ANOVA y Bonferroni. Finalmente, los datos fueron procesados para el establecimiento de relaciones entre variables y el análisis de las diferencias en apoyo social y recursos afectivos en grupos de alta y baja vulnerabilidad.

\section{Resultados}

La VCD se encontró presente en una buena proporción de sujetos estudiados (62\% de la población). De éstos, el 55.17\% (32) presentó baja VCD mientras que el resto, el 44.83\% (26), presentó alta VCD.

El análisis realizado para observar la distribución de los estudiantes con y sin VCD en las categorías de depresión mayor evaluadas por la CES-DR indicó que el $92.47 \%$ de la población (86 participantes) se ubicó en un nivel de no significancia clínica, éstos se concentraron en los distintos grupos como sigue: $38 \%$ en el grupo sin VCD, 34\% en el grupo de baja VCD, y el $28 \%$ en el de alta VCD. Estos resultados indican que aunque la mayor parte de la población no presentó sintomatología clínicamente significativa para un episodio depresivo mayor, en contraparte sí mostraron vulnerabilidad cognitiva a la depresión. Cabe resaltar que de los casos restantes, 2 se observaron en EDM, otro en probable EDM y uno más en alta probabilidad de EDM; todos ellos, presentaron VCD (ver Tabla 1). 
Tabla 1.

Distribución de la población con y sin VCD en las categorías clínicas de EDM evaluadas por la CES-D-R (35)

\begin{tabular}{lrrrr}
\hline \multirow{2}{*}{ Criterios $^{\mathrm{a}}$} & \multicolumn{3}{c}{ VCD } & \multirow{2}{*}{ Total } \\
\cline { 2 - 4 } & sin & baja & alta & \\
\hline Sin relevancia clínica & 33 & 29 & 24 & 86 \\
Sub-umbral de depresión & 1 & 1 & 0 & 2 \\
Probable EDM & 0 & 0 & 1 & 1 \\
Alta probabilidad de EDM & 1 & 1 & 0 & 2 \\
EDM & 0 & 1 & 1 & 2 \\
\hline Total & 35 & 32 & 26 & 93 \\
\hline
\end{tabular}

${ }^{a}$ Criterios (DSM-IV) para EDM evaluados con la CES-D-R (35).

En términos generales los resultados del análisis de correlación muestran asociaciones significativas entre las variables apoyo social, recursos psicológicos y vulnerabilidad cognitiva a la depresión (Tabla 2). Los puntajes de desesperanza y depresión arrojados por la CES-DR no observaron relación entre la red social y los recursos psicológicos pero sí con la VCD. La red de amigos presentó una correlación negativa débil, aunque signifi-cativa, con los recursos psicológicos de red de apoyo $(r=-.329, p \leq .01)$ y de autocontrol $(r=-.334$, $p \leq .01)$; de igual forma presentó una correlación positiva moderada con la red de compañeros/vecinos $(r=.549$, $p \leq .01)$ y una correlación positiva débil con la VCD $(r=.259$, $p \leq .05)$. 
Tabla 2.

Correlaciones entre apoyo social, recursos psicológicos, VCD, desesperanza y depresión

\begin{tabular}{|c|c|c|c|c|c|c|c|c|c|c|c|}
\hline \multicolumn{12}{|c|}{ Variables } \\
\hline & 1 & 2 & 3 & 4 & 5 & 6 & 7 & 8 & 9 & 10 & 11 \\
\hline \multicolumn{12}{|c|}{ Apoyo social } \\
\hline $\begin{array}{l}\text { 1. Amistad/ } \\
\text { confianza }\end{array}$ & - & $.549^{* *}$ & $-329^{* \star *}$ & .198 & $-334^{* *}$ & .042 & -134 & 198 & $.259 *$ & .041 & .005 \\
\hline $\begin{array}{l}\text { 2. Compañeros/ } \\
\text { vecinos }\end{array}$ & & - & $-238^{* \star}$ & $.297^{* \star}$ & $-310^{* *}$ & .173 & -186 & 198 & $.324^{* *}$ & .021 & .070 \\
\hline
\end{tabular}

\section{Recursos psicológicos}

3. Red de apoyo

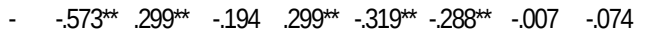

4. Incapacidad p. solicitar apovo

$\begin{array}{llllllll}- & -.227^{\star} & .391^{* *} & -.190 & .285^{* *} & .465^{* *} & .061 & .197\end{array}$

5. Autocontrol

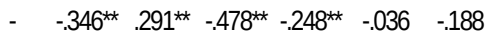

6. Dificultad maneio de tristeza

7. Recuperación del equilibrio

8. Manejo del enoio

Vulnerabilidad y depresión

9. VCD

10. Desesperan-

$\mathrm{za}$

11. Depresión

${ }^{*} p \leq .05$

${ }^{\star *} p \leq .01$

La VCD por su parte, mostró correlaciones positivas débiles con la red de compañeros y vecinos 
( $r=.324, p \leq .01)$, el manejo del enojo $(r=.244, p \leq .05)$, y dificultad en el manejo de la tristeza $(r=.397, p \leq .001)$, y moderada con la incapacidad para solicitar apoyo $(r=.465, p \leq .01)$. Correlacionó negativamente, aunque de manera débil, con los recursos psicológicos de red de apoyo $(r=-.288, p \leq .01)$ y autocontrol $(r=-.248, p \leq .01)$. Finalmente, la depresión mostró una correlación positiva relativamente fuerte con desesperanza $(r=.696, p \leq .05)$ y una correlación débil con VCD ( $r=204, p \leq .05)$. Lo anterior sugiere que los sujetos que tienen VCD pueden presentar síntomas de desesperanza y de depresión.

Se encontró que entre los grupos con y sin VCD existen diferencias estadísticamente significativas en: a) la red de apoyo de compañeros y vecinos, llamando la atención que el grupo con alta VCD es el que mejor percibe tener relaciones cordiales y amistosas con compañeros y vecinos cuando se tienen dificultades $(\mathrm{G} 1=2.35, \mathrm{G} 2=2.13$, $\left.\mathrm{G} 3=2.48, F=3.211_{(2,89)}, p=.045\right) ;$ b) red de apoyo (G1 = $\left.\left.3.50, \mathrm{G} 2=3.59, \mathrm{G} 3=3.20, F=3.255_{(2,90)}, p=.043\right) ; \mathrm{c}\right)$ dificultad para solicitar apoyo (G1 = 2.06, G2 = 1.58, G3 = 2.30, $\left.\left.F=9.021_{(2,90)}, p=.000\right) ; d\right)$ autocontrol (G1 = 3.50, G2 $\left.=3.36, \mathrm{G} 3=3.14, F=4.401_{(2,89)}, p=.015\right)$; e) dificultad en el manejo de la tristeza $(\mathrm{G} 1=2.18, \mathrm{G} 2=1.76, \mathrm{G} 3=2.32, F=$ $\left.8.545_{(2,89),} p=.000\right) ; \mathrm{y}$ f) dificultad en el manejo del enojo (G1 $\left.=1.60, \mathrm{G} 2=1.68, \mathrm{G} 3=2.04, F=5.345_{(2,89)}, p=.006\right)$.

La dificultad para solicitar apoyo, dificultad para mane-jar la tristeza y dificultad en el manejo del enojo presentaron diferencias estadísticamente significativas. La depresión no resultó tener una diferencia estadística 
significativa entre los grupos al igual que la red social de amigos y el recurso de recuperación del equilibrio (Tabla 3).

Tabla 3.

ANOVA para los grupos con y sin vulnerabilidad cognitiva a la depresión

\begin{tabular}{|c|c|c|c|c|c|c|}
\hline \multirow{2}{*}{ Escala } & \multicolumn{3}{|c|}{ VCD } & \multirow{2}{*}{$\mathrm{F}$} & \multirow{2}{*}{ GL } & \multirow{2}{*}{$p$} \\
\hline & $\sin$ & baja & alta & & & \\
\hline \multicolumn{7}{|c|}{ Depresión } \\
\hline Total CES-D-R & 1.66 & 1.66 & 1.83 & 1.327 & 2,90 & .271 \\
\hline \multicolumn{7}{|c|}{ Apoyo social } \\
\hline $\begin{array}{l}\text { Amistad/ } \\
\text { Confianza }\end{array}$ & 1.63 & 1.62 & 1.85 & 1.944 & 2,89 & 150 \\
\hline $\begin{array}{l}\text { Compañeros/ } \\
\text { Vecinos }\end{array}$ & 2.35 & 2.13 & 2.48 & 3.211 & 2,89 & .045 \\
\hline \multicolumn{7}{|c|}{ Recursos psicológicos } \\
\hline Red de Apoyo & 3.50 & 3.59 & 3.20 & 3.255 & 2,90 & .043 \\
\hline $\begin{array}{l}\text { Dificultad para } \\
\text { solicitar apovo }\end{array}$ & 2.06 & 1.58 & 2.30 & 9.021 & 2,90 & .000 \\
\hline Autocontrol & 3.50 & 3.36 & 3.14 & 4.401 & 2,89 & .015 \\
\hline $\begin{array}{l}\text { Dificultad mane- } \\
\text { io de tristeza }\end{array}$ & 2.18 & 1.76 & 2.32 & 8.545 & 2,89 & .000 \\
\hline $\begin{array}{l}\text { Recuperación } \\
\text { del equilibrio }\end{array}$ & 3.09 & 3.15 & 2.88 & 1.027 & 2,90 & .362 \\
\hline $\begin{array}{l}\text { Manejo del } \\
\text { enoio }\end{array}$ & 1.60 & 1.68 & 2.04 & 5.345 & 2,89 & .006 \\
\hline
\end{tabular}


Como se observa en la Tabla 4 el análisis Bonferroni mostró que las diferencias se presentan específicamente entre los grupos con VCD.

Tabla 4.

Comparación múltiple entre los grupos sin y con VCD (alta y baja) con ajuste de Bonferroni

\begin{tabular}{lcccc}
\hline \multicolumn{1}{c}{ Variable } & $\begin{array}{c}\text { Grupos que explican la } \\
\text { diferencia }\end{array}$ & $\begin{array}{c}\text { Dif. } \\
\text { medias }\end{array}$ & $\begin{array}{c}\text { Error } \\
\text { típico }\end{array}$ & $p$ \\
\hline $\begin{array}{l}\text { 1.Compañeros/ } \\
\text { Vecinos }\end{array}$ & VCD BAJA - VCD ALTA & -.347 & .140 & .046 \\
2.Red de Apoyo & VCD BAJA - VCD ALTA & -.393 & .159 & .046 \\
& VCD BAJA - VCD ALTA & .727 & .177 & .000 \\
$\begin{array}{l}\text { 3.Dificultad para } \\
\text { solicitar apoyo }\end{array}$ & SIN VCD - VCD BAJA & .487 & .164 & .011 \\
$\begin{array}{l}\text { 4.Autocontrol } \\
\text { SIN VCD - VCD ALTA }\end{array}$ & .362 & .122 & .012 \\
$\begin{array}{l}\text { 5.Dificultad } \\
\text { manejo de } \\
\text { tristeza }\end{array}$ & VCD BAJA - VCD ALTA & -.554 & .142 & .001 \\
$\begin{array}{l}\text { 6.Dificultad en el } \\
\text { manejo del } \\
\text { enojo }\end{array}$ & VCD BAJA - VCD ALTA & -.360 & .143 & .041 \\
\hline & SIN VCD - VCD ALTA & -.442 & .140 & .007 \\
\hline
\end{tabular}

\section{Discusión}

Los resultados sugieren que las redes de amigos, compañeros y vecinos no mostraron tener relación con la sintomatología depresiva o la desesperanza. Estos 
dos tipos de apoyo social, parecen tener una relación más directa y positiva con la vulnerabilidad cognitiva a la depresión y los componentes de atribución de causalidad y consecuencias. Estos hallazgos son similares a los reportados por Lamis, Ballard, May y Dvorak (2016) y Panzarella, Alloy y Whitehouse (2006), dado que el apoyo social no presenta una relación directa con los síntomas depresivos puede pensarse como una variable mediadora.

Un estudio similar realizado por Bisschop (2004) determinó el impacto que sobre la sintomatología depresiva tiene el tamaño de la red, el estatus del compañero, el apoyo instrumental, y el apoyo emocional, utilizando la CES-D. Otros estudios muestran evidencia contraria a la encontrada en este estudio y reportan relaciones directas del apoyo social con la sintomatología depresiva; por ejemplo, los hallazgos de Nava al analizar la relación entre la red social y la depresión con adolescentes mostraron que la red de amigos medida a través de la ECAR correlacionó de manera moderada con depresión; asimismo, el estudio realizado con jóvenes estadounidenses para analizar la relación entre síntomas de depresión y apoyo social en la transición a la adultez joven en donde altos niveles de sintomatología depresiva se asociaron con menor apoyo de los padres durante la adultez joven.

Esta contradicción en los resultados nos deja ver que el rol que juega el apoyo social en la presencia de sintomatología depresiva es confuso. El concepto es 
tan ambiguo que las futuras investigaciones deben avocarse al estudio del impacto de las redes de apoyo social con la mayor especificidad posible en el manejo del concepto.

Por lo que respecta a los recursos psicológicos mostraron relacionarse con la vulnerabilidad cognitiva a la depresión y con la red de apoyo social.

Así pues los resultados encontrados sugieren que la asociación encontrada entre apoyo social y VCD indican que los jóvenes universitarios con vulnerabilidad cognitiva a la depresión perciben tener relaciones de amistad fuertes y duraderas, basadas en la reciprocidad, conocimiento, comprensión, confianza y la convivencia compartida; sin embargo, son los sujetos que les es muy difícil pedir ayuda a los demás, no saben a quién recurrir ante una situación grave, se sienten avergonzados al pedir ayuda y evitan a los demás cuando tienen un problema; asimismo, cuando se enojan pierden el control, se alteran, pierden la tranquilidad y la compostura. En contraparte, los sujetos con baja vulnerabilidad cognitiva perciben tener alta capacidad para solicitar ayuda y perciben tener al menos una persona con la que pueden contar; saben manejar de manera adecuada la tristeza (perciben solución a sus problemas, el desánimo no llega tan fácilmente, se les facilita recuperar la tranquilidad, recuperan con facilidad su bienestar ante la frustración y tienen la capacidad para no permitir que el sentimiento de tristeza se incremente). Aunque estos 
resultados resultan contradictorios con otros estudios nos hacen reflexionar en la necesidad de seguir estudiando estas relaciones a fin de comprender la dinámica del origen y mantenimiento de la depresión en los jóvenes.

\section{Referencias}

Beiter, R., Nash, R., McCrady, M., Rhoades, D., Linscomb, M., Clarahan, M., \& Sammut, S. (2015). The prevalence and correlates of depression, anxiety, and stress in a sample of college students. Journal of Affective Disorders, 173, 9096. doi:10.1016/j.jad.2014.10.054

Benjet, C., Borges, G., Méndez, E., Albor, Y.,

Casanova, L., Orozco, R., Curiel, T., Fleiz, C., \& Medina-Mora, M. E. (2016). Eight-year incidence of psychiatric disorders and service use from adolescence to early adulthood: longitudinal follow-up of the Mexican Adolescent Mental Health Survey. Europe Child Adolescense Psychiatry, 25(2), 163-73.

Benjet, C., Bromet, E., Karam, E. G., Kessler, R. C., McLaughlin, K. A., Ruscio, A.M., Shahly, V., Stein, D. J., Petukhova, M., Hill, E., Alonso, J., Atwoli, L., Bunting, B., Bruffaerts, R., Caldas-de-Almeida, J. M., de Girolamo, G., Florescu, S., Gureje, O., Huang, Y., Lepine, J. P, Kawakami, N., KovessMasfety, V., Medina-Mora, M. E., Navarro-Mateu, 
F., Piazza, M., Posada-Villa, J., Scott, K. M., Shalev, A., Slade, T., ten Have, M., Torres, Y., Viana, M. C., Zarkov, Z., \& Koenen, K. C. (2016).

The epidemiology of traumatic event exposure worldwide: results from the World Mental the World Mental Health Survey Consortium.

Psychology Medical, 46(2), 327-43.

Benjet, C., Medina-Mora, M.E.; Borges, G.; Martínez, M.K., Hermosillo_de la Torre, A.E.; Gutiérrez, R.; Albor,Y.; Lara, C.; Auerbach, R.; Bruffaerts, R.;

Cuijpers, R.; Green, J. \& Kessler, R. (2016).

Primer informe de los alumnos de nuevo ingreso 2016 de la Universidad Autónoma de

Aguascalientes. Proyecto Universitario para alumnos saludables PUERTAS.

Eaton, W., Muntaner, C., \& Smith, C. (1998). Revision of the Center for Epidemiologic Studies Depression (CES-D) Scale. Baltimore: Johns Hopkins University.

Ferrari, A. J., Norman, R. E., Freedman, G., Baxter, A. J., Pirkis, J. E., Harris, M. G. \& Whiteford, H. A. (2014). The Burden Attributable to Mental and Substance Use Disorders as Risk Factors for Suicide: Findings from the Global Burden of Disease Study 2010. PLoS One, 9(4), e91936. Gómez-Restrepo, C. Bohórquez, A., Pinto, M. D., GilLaverde, J. F. A., Rondón, S. M., \& DíazGranados, N. (2004). Prevalencia de depresión y factores asociados con ella en la población 
colombiana. Revista Panamericana de Salud

Pública; 16(6), 378-86.

Mac Giollabhui, N., Hamilton, J. L., Nielsen, J.,

Connolly, S. L., Stange, J. P., Varga, S., Burdette,

E., Olino, T. M., Abramson, L. Y., \& Alloy, L. B.

(2018). Negative cognitive style interacts with

negative life events to predict first onset of a major

depressive episode in adolescence via

hopelessness. Journal of Abnormal Psychology,

127(1), 1-11.

Nava, Q.C., \& Vega, V.C. (2008). Dinámica de la red social y alteración psicológica en adolescentes con ausencia de familia de origen. Revista Diversas Perspectivas en Psicología, 4(1), 417425.

Needham, B. L. (2008). Reciprocal relationships between symptoms of depression and parental support during the transition from adolescence to young adulthood. Journal of Youth Adolescence, 37(1), 893-905.

Panzarella, C., Alloy, L. B., \& Whitehouse, W. G. (2006).

Expanded Hopelessness Theory of Depression:

On the mechanisms by which social support protects against depression. Cognitive Therapy and Research, 30, 307-333. doi:10.1007/s10608006-9048-3

Wagner, F., Gallo, J., \& Delva, J. (1999). Depression in late life: A hidden public health problem for Mexico? Salud Pública México, 41, 189-202,. 
World Health Organization (2017). Depression and Other Common Mental Disorders: Global Healt Estimates. Geneva: WHO

Xiao, J., Qiu, Y., He, Y., Cui, L., Auerbach, R. P., McWhinnie, C. M., \& Yao, S. (2016). "Weakest

Link" as a Cognitive Vulnerability within the Hopelessness Theory of Depression in Chinese University Students. Stress and Health : Journal of the International Society for the Investigation of Stress, 32(1), 20-27. doi:10.1002/smi.2571

\section{Agradecimientos}

Queremos externar un agradecimiento muy especial al Dr. Fernando Wagner por su valiosa colaboración y ayuda con el procesamiento de los datos de la CES-DR 35 obtenidos para la realización de este estudio; asimismo al Dr. José Filiberto Enríquez Bielma y la Dra. Verónica Ortiz Lefort por su asesoría y apoyo. 


\section{(c) (1) (9)(2)}

\section{Esta obra está bajo una \\ Licencia Creative Commons Atribución-NoComercial- Compartirlgual 4.0 Internacional}

Usted es libre de compartir o adaptar el material en cualquier medio o formato bajo las condiciones siguientes: (a) debe reconocer adecuadamente la autoría, proporcionar un enlace a la licencia e indicar si se han realizado cambios; (b) no puede utilizar el material para una finalidad comercial y (c) si remezcla, transforma o crea a partir del material, deberá difundir sus contribuciones bajo la misma licencia que el original.

Resumen de la licencia https://creativecommons.org/licenses/by-nc-sa/4.0/deed.es_ES

Texto completo de la licencia https://creativecommons.org/licenses/by-nc-sa/4.0/legalcode 